\title{
Duration of RT-PCR positivity in severe acute respiratory syndrome
}

\author{
C.M. Chu*, W.S. Leung*, V.C.C. Cheng", K.H. Chan\#, A.W.N. Lin*, V.L. Chan*, \\ J.Y.M. Lam*, K.S. Chan* and K.Y. Yuen\#
}

ABSTRACT: Severe acute respiratory syndrome (SARS) is a highly infectious respiratory infection with a high mortality. The duration of infectivity is unknown. The RT-PCR positivity for SARSassociated coronavirus (SARS-CoV) was followed in $\mathbf{4 5}$ virologically confirmed SARS patients.

Serial RT-PCRs for SARS-CoV were performed in the nasopharyngeal aspirate, stool and urine of 45 SARS patients who survived until discharge. All patients had at least one site that was positive for SARS-CoV on presentation. Time to RT-PCR conversion was studied in all patients.

There were 15 males (33.3\%) and 30 females (66.7\%), with a mean \pm SD age of $40.7 \pm 14.7$ yrs. The median (range) time of RT-PCR conversion was 30 days (2-81). On discharge from the hospital, 18 (40\%) remained RT-PCR positive in at least one site. For patients with positive RT-PCR on discharge, the median (range) time to RT-PCR conversion after discharge was 13 days (2-60).

A significant proportion of severe acute respiratory syndrome patients remained RT-PCR positive for severe acute respiratory syndrome-associated coronavirus for a substantial duration after discharge. The clinical significance is unknown and this finding merits further study. It is prudent to advise patients to adhere to strict personal hygiene on discharge until RT-PCR becomes negative.

KEYWORDS: Infectivity, RT-PCR, severe acute respiratory syndrome, severe acute respiratory syndrome-associated coronavirus, transmission

evere acute respiratory syndrome (SARS) is a highly infectious, rapidly progressive pneumonia affecting all age sectors. SARS has spread in an epidemic manner. The total number of cases worldwide has reached $>8,000$ with 774 mortalities within a period of 9 months [1]. The average number of secondary cases generated by a "typical" infectious individual (basic reproductive number, R0) in a completely susceptible population, in the absence of control measures, was estimated to be around three for SARS [2-4]. The duration of infectivity is currently unknown. The World Health Organization (WHO) advises that patients with SARS can be considered for discharge from hospital if they are afebrile for $48 \mathrm{~h}$ with resolving cough, together with improving radiological, haematological and biochemical parameters [5]. However, transmission to close contacts by recovered SARS patients was reported [6]. In this study, the duration of positivity of RT-PCR to SARS-associated coronavirus (SARS-CoV) was examined in clinical specimens of patients with SARS.

\section{MATERIALS AND METHODS}

This is a retrospective analysis of prospectively collected clinical and virological data of patients with confirmed SARS, admitted to the Dept of Medicine and Geriatrics, United Christian Hospital, Hong Kong SAR, China, during a community outbreak from March 24 to May 4, 2003. All patients met a modified WHO definition of SARS, which included fever of $\geqslant 38^{\circ} \mathrm{C}$, cough or shortness of breath, new pulmonary infiltrates on radiological examination, and absence of an alternative diagnosis, together with virological documentation of SARS-CoV infection (paired serology and/or positive RT-PCR for SARS-CoV from clinical specimens). All patients were treated with a standard protocol of broadspectrum antibiotics, ribavirin and a tailing regimen of corticosteroid [7, 8]. A proportion of patients were treated additionally with an experimental agent, lopinavir/ritonavir (LPV/r) in an open-label clinical trial [9]. The clinical, haematological, biochemical, radiological and virological findings were prospectively entered into a

\section{AFFILIATIONS}

*Division of Respiratory Medicine, Dept of Medicine and Geriatrics, United Christian Hospital, and

\# Dept of Microbiology, Queen Mary Hospital, The University of Hong Kong, Hong Kong SAR, China.

\section{CORRESPONDENCE}

C.M. Chu

Division of Respiratory Medicine Dept of Medicine and Geriatrics United Christian Hospital Hong Kong SAR

China

Fax: 85235135548 E-mail: chucm@ha.org.hk

Received:

May 142004

Accepted:

August 162004

SUPPORT STATEMENT

This study was supported by research funding from the Vice-Chancellor SARS Research Fund, Hong Kong SAR, China. 
pre-set database, and the findings in the first 75 patients had been reported previously [8].

Nasopharyngeal aspirate (NPA), urine and stool specimens were collected on presentation and RT-PCR was performed according to previous publications $[8,10]$. Patients who had positive RT-PCR in NPA, stool or urine at presentation were included. In the included patients, follow-up specimens were taken at intervals of $7 \pm 2$ days from symptom onset, until SARS-CoV was undetectable in two consecutive specimens. Patients who died during hospitalisation were excluded from the final analysis.

Data are expressed as mean $\pm \mathrm{SD}$, unless otherwise specified. Time to RT-PCR conversion was calculated from the date of specimen collection on presentation to the day when SARS$\mathrm{CoV}$ was undetectable from all clinical specimens, which had to be confirmed by consecutive negative tests from all clinical sites. The potential associations of the following baseline characteristics with the time to RT-PCR conversion were explored: age, sex, active comorbid conditions, sites of positive RT-PCR, the need for pulse methylprednisolone rescue, diarrhoea, first lactate dehydrogenase level, initial viral load of NPA specimens, and whether the patient was treated with $\mathrm{LPV} / \mathrm{r}$. Potential risk factors affecting the time to RT-PCR conversion were analysed by the log-rank test (for categorical variables) and Cox's regression (for continuous variables). Hazard ratios (HRs) are given with 95\% confidence interval (CI). The Kaplan-Meier product-limit estimator was used to estimate RT-PCR conversion and for the time-to-conversion plot. A two-tailed p-value of $<0.05$ was considered statistically significant.

\section{RESULTS}

There were 127 patients with confirmed SARS admitted to the current authors' unit during the study period, of whom 52 $(40.9 \%)$ had a positive RT-PCR from NPA, stool or urine at the time of presentation. Of these 52 patients, seven died during hospitalisation, and they were excluded from further analysis. A remaining 45 patients were available for study, and followup was complete in all of them.

Of the 45 patients, the mean \pm SD age was $40.7 \pm 14.7 \mathrm{yrs}$, with $15(33.3 \%)$ males and $30(66.7 \%)$ females. They presented at a median of 3 days from symptom onset. All had virological confirmation by RT-PCR in at least one clinical site, together with positive paired serology. In these 45 patients, the specimens in which RT-PCR was positive on presentation were as follows: NPA in $36(80 \%)$, stool in $38(84.4 \%)$ and urine in 15 $(33.3 \%)$. All three sites were positive in 13 patients $(28.9 \%)$, and two sites were positive in 18 patients $(40 \%)$. The patients stayed a mean \pm SD of $35.2 \pm 15.4$ days in hospital. The median (range) time of RT-PCR conversion was 30 days (2-81; fig. 1). On discharge from the hospital, $18(40 \%)$ remained RT-PCR positive in at least one site. NPA was positive in $10(22.2 \%)$, stool in seven $(15.6 \%)$ and urine in five $(11.1 \%)$ patients. All three sites were positive in one patient $(2.2 \%)$ and two sites were positive in two $(4.4 \%)$. The median (range) time to RTPCR conversion from the day of discharge was 13 days (2-60).

Positive initial urine RT-PCR was predictive of a delay in RT-PCR conversion ( $p=0.0077$; fig. 2 ). Patients who received $\mathrm{LPV} / \mathrm{r}$, in addition to standard treatment with ribavirin and

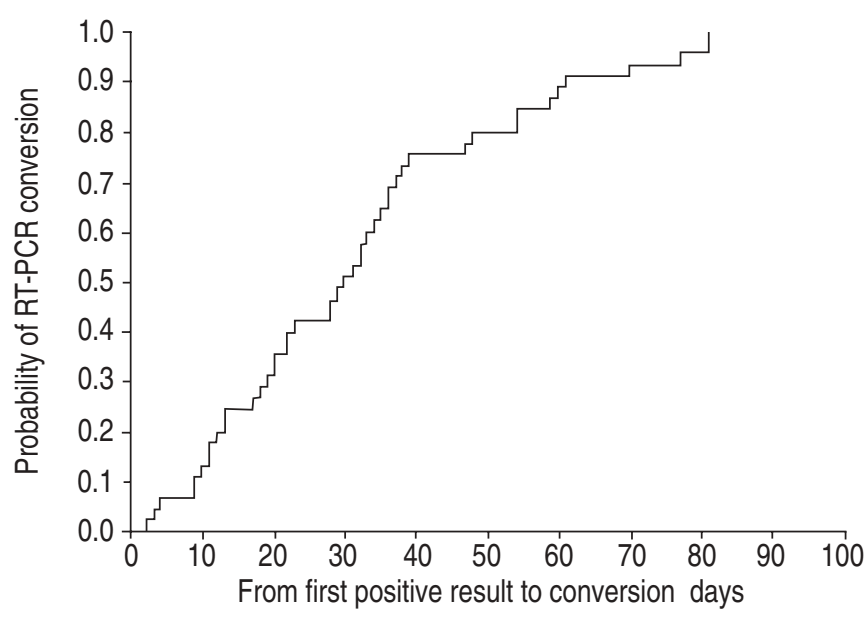

FIGURE 1. Kaplan-Meier curve showing the time for RT-PCR conversion from the first positive specimen.

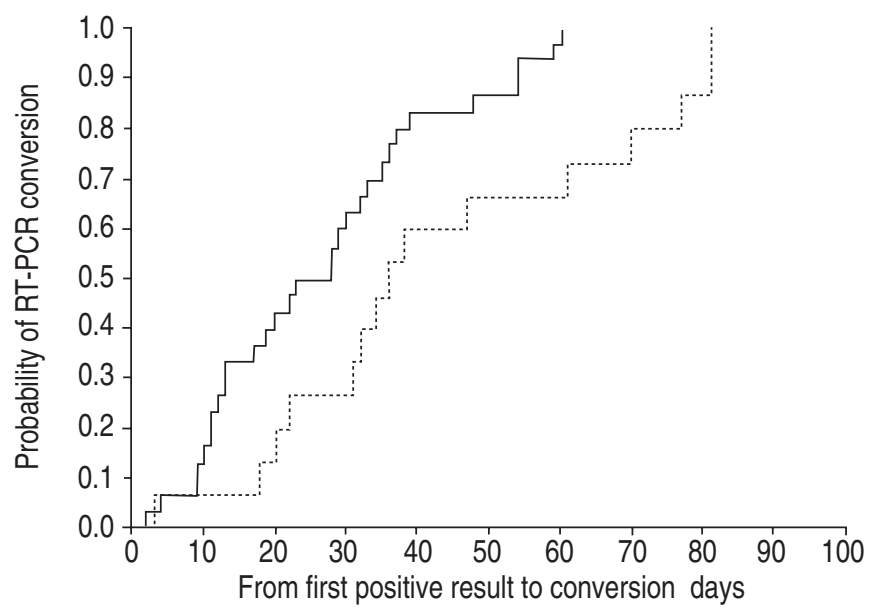

FIGURE 2. Time to RT-PCR conversion with respect to the initial urine RT-PCR status: $\cdots \cdots$ : positive $(n=15)$; —

corticosteroid, had earlier RT-PCR conversion $(p=0.0281$; fig. 3). Multivariate Cox's regression showed both factors were independently associated with time to RT-PCR conversion. The adjusted HRs for urine RT-PCR positivity and LPV $/ \mathrm{r}$ treatment were 0.39 (95\% CI: 0.19-0.82; $\mathrm{p}=0.013)$ and $1.96(95 \%$ CI: 1.02-3.75; $\mathrm{p}=0.043)$, respectively. The other baseline factors were not predictive of the time to RT-PCR conversion.

\section{DISCUSSION}

This study shows that a significant proportion of SARS patients have detectable viral RNA for SARS-CoV in their clinical specimens for a substantial period of time. Positive urine RT-PCR on presentation is related to delayed RT-PCR conversion, whilst treatment with $\mathrm{LPV} / \mathrm{r}$ is associated with earlier conversion. It is noteworthy that the current patients had already stayed for a mean of 35.2 days in hospital before discharge, and yet a significant proportion (40\%) still had at least one site that was RT-PCR positive at the time of discharge. The prolonged hospital stay was due to a local quarantine policy that demanded SARS patients to remain in 


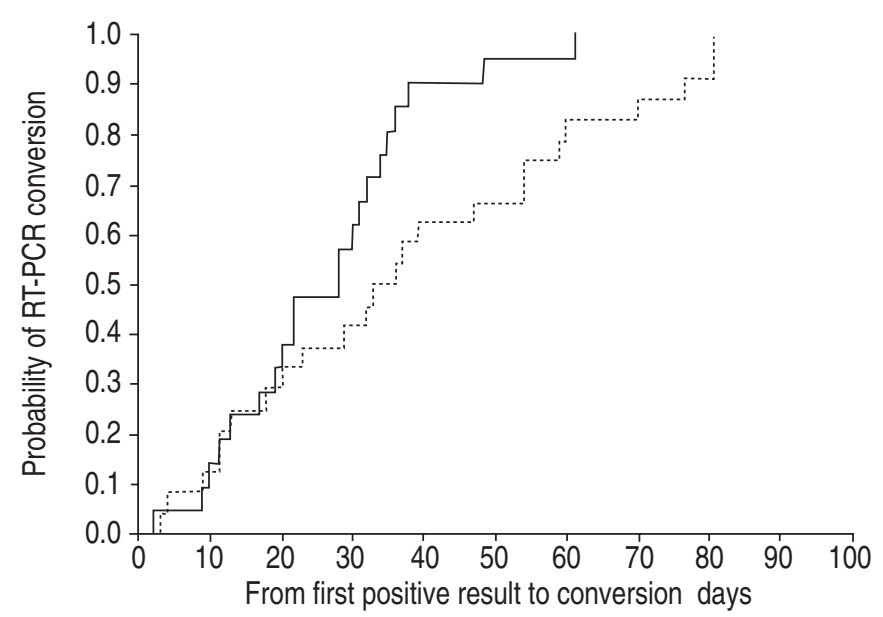

FIGURE 3. Time to RT-PCR conversion with respect to the treatment received

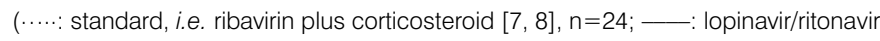
in addition to standard treatment [9], $n=21) . p=0.0281$.

hospital until they met the WHO discharge criteria [5], or for 21 days, whichever was longer.

The findings of this study agree with a laboratory-based study that viral RNA may still be detectable in clinical specimens in some patients $>30$ days from symptom onset [11]. It is interesting to find that urine RT-PCR positivity was related to delayed RT-PCR conversion. SARS is predominantly a disease of the aerodigestive tract and SARS-CoV is mostly recovered from the nasopharynx and the stool, and the virus is less frequently detectable in the urine $[11,12]$. Positive urine RT-PCR for SARS-CoV may reflect a higher viral load and/or the susceptibility for dissemination in an individual, with resulting delayed clearance of the virus. Treatment with $\mathrm{LPV} / \mathrm{r}$ was associated with earlier RT-PCR conversion, and this finding is consistent with the current authors' previous report that $\mathrm{LPV} / \mathrm{r}$ was associated with lowering of viral load during the course of illness and improved clinical outcomes when compared with historical controls [9].

The clinical significance of the prolonged RT-PCR positivity is currently unknown. Although viral RNA remains detectable in clinical specimens, it does not imply that the virus is necessarily viable or transmissible. No transmission to close contacts was documented in the current patients after discharge. However, it should be noted that the current patients were advised to exercise strict personal hygiene measures until RT-PCR conversion was confirmed. Conversely, SARS-CoV is difficult to culture from clinical specimens. Virus isolation from various clinical specimens ranged only $21.4-37.9 \%$ during the first 2 weeks of illness, despite the high infectivity during this period [11]. Negative culture cannot be taken to mean zero risk of infectivity. Moreover, transmission to close contacts by recovered SARS patients is known to have occurred [6]. Before there is further data to ascertain the duration of infectivity in SARS, the current authors feel that it is prudent to monitor RTPCR status in various clinical specimens of SARS patients.
SARS patients should also be advised of hygienic measures (e.g. wearing a surgical mask, toilet disinfection, disinfection of food utensils) until RT-PCR conversion is confirmed.

To conclude, a significant proportion of severe acute respiratory syndrome patients remained RT-PCR positive for severe acute respiratory syndrome-associated coronavirus on discharge for a substantial period of time. Further studies are needed to explore the duration of infectivity in severe acute respiratory syndrome patients with delayed RT-PCR conversion.

\section{REFERENCES}

1 World Health Organization. Summary of probable SARS cases with onset of illness from 1 November 2002 to 31 July 2003. www.who.int/csr/sars/country/table2004_04_21/ en/. Date last updated: April 21 2004; Date last accessed: October 222004.

2 Riley S, Fraser C, Donnelly CA, et al. Transmission dynamics of the etiological agent of SARS in Hong Kong: impact of public health interventions. Science 2003; 300: 1961-1966.

3 Lipsitch M, Cohen T, Cooper B, et al. Transmission dynamics and control of severe acute respiratory syndrome. Science 2003; 300: 1966-1970.

4 Lloyd-Smith JO, Galvani AP, Getz AP. Curtailing transmission of severe acute respiratory syndrome within a community and its hospital. Proc R Soc Lond B Biol Sci 2003; 270: 1979-1989.

5 World Health Organization. WHO hospital discharge and follow-up policy for patients who have been diagnosed with severe acute respiratory syndrome (SARS). www. who.int/csr/sars/discharge/en. Date last updated: March 28 2003; Date last accessed: October 222004.

6 Chan LY, Li PK, Sung J. Risk of SARS transmission to persons in close contact with discharged patients. Am J Med 2003; 115: 330.

7 So LK, Lau AC, Yam LY, et al. Development of a standard treatment protocol for severe acute respiratory syndrome. Lancet 2003; 361: 1615-1617.

8 Peiris JS, Chu CM, Cheng VC, et al. Clinical progression and viral load in a community outbreak of coronavirusassociated SARS pneumonia: a prospective study. Lancet 2003; 361: 1767-1772.

9 Chu CM, Cheng VC, Hung IF, et al. Role of lopinavir/ ritonavir in the treatment of SARS: initial virological and clinical findings. Thorax 2004; 59: 252-256.

10 Poon LL, Wong OK, Chan $\mathrm{KH}$, et al. Rapid diagnosis of a coronavirus associated with severe acute respiratory syndrome (SARS). Clin Chem 2003; 49: 953-955.

11 Chan KH, Poon LL, Cheng VC, et al. Detection of SARS coronavirus in patients with suspected SARS. Emerg Infect Dis 2004; 2: 294-299.

12 Cheng VC, Hung IF, Tang BS, et al. Viral replication in the nasopharynx is associated with diarrhea in patients with severe acute respiratory syndrome. Clin Infect Dis 2004; 38: 467-475. 